\title{
El comportamiento de los empleadores ante el fenómeno de la temporalidad
}

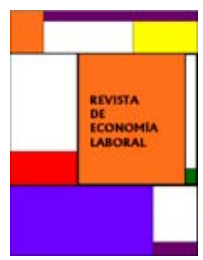

\author{
Anna Laborda ${ }^{1}$ \\ ESADE Business School
}

Recibido el 21 de septiembre de 2007; aceptado el 20 de mayo de 2008

\section{Resumen}

El objetivo del presente estudio es el de establecer hipótesis sobre las motivaciones de los empleadores a utilizar la contratación temporal. Como resultado, formulo diversas hipótesis de trabajo que ayudarían a explicar la "cultura de la temporalidad". Las posibles variables explicativas serían: antigüedad e historia de la empresa; relación del lugar de trabajo con el cliente final; actividad regular vs. irregular, independientemente del sector, y nivel de competencia del mercado en el que actúa la empresa. El objetivo de próximos estudios será validar dichas hipótesis.

Palabras clave: Temporalidad, modelos por etapas, modelo transteorético, comportamiento de los empleadores.

Clasificación JEL: J23, J50, M12, A12

\begin{abstract}
This study aims to establish hypotheses regarding employers' motivations for hiring temporary workers. As a result, I have formulated various working hypotheses which would help explain the "temp culture". The possible explanatory variables would be: seniority and company history; workplace / end client relationship; regular vs. irregular employment, regardless of the sector, and the company's market competition level. Validating these hypotheses will be the objective of future studies.
\end{abstract}

Keywords: Fixed-term contracts, stages models, transtheoretical model, employers' behaviour.

JEL Classification: J23, J50, M12, A12

${ }^{1}$ Dirección para correspondencia: anna.laborda@esade.edu. Este trabajo se presentó en las VII Jornadas de Economía Laboral celebradas en Gran Canaria en julio de 2007.

(C) Revista de Economía Laboral 


\section{Introducción}

A pesar de que existen numerosos estudios sobre el fenómeno de la temporalidad en España, todos de gran calidad ${ }^{2}$, lo cierto es que son todavía muchas las lagunas por llenar, y quizá la razón de ello sea que la información disponible no alcanza a cubrir todas las cuestiones relevantes para la explicación del problema. Este lag de información podría darse en más de un aspecto, pero el que creo que presenta mayor interés para el avance en la comprensión del fenómeno es el del comportamiento de los empleadores.

Es cierto que la ley establece el marco en el cual se permiten o no se permiten ciertos tipos de comportamientos, y que parece que el nuevo marco legal está incentivando la contratación indefinida y la conversión de contratos temporales a indefinidos, pero ¿los incentivos suministrados provocarán un verdadero cambio de comportamiento de las personas que toman las decisiones de contratación? Para resolver estas cuestiones me parece de gran interés dedicar parte del esfuerzo investigador en recabar información que provenga directamente de los empleadores y que nos permita dar respuesta a la pregunta "¿por qué los empleadores utilizan el contrato temporal?", respuesta que considero esencial para la comprensión de la situación.

\section{Objetivo y metodología}

El objetivo del presente estudio es el de conocer las prácticas utilizadas en la contratación y averiguar cuáles son las motivaciones que inducen al uso de la contratación temporal como forma de relación laboral por parte de los empleadores.

La información incluida en estas notas de investigación se ha obtenido utilizando la metodología de la "entrevista personal". Esta metodología es adecuada para obtener un primer conjunto de información, que conformaría los resultados de una investigación cualitativa $^{3}$, que son los que se presentan en este trabajo ${ }^{4}$. La

\footnotetext{
2 Ver, entre otros, Aguirregabiria y Alonso-Borrego (1999); Dolado et al. (2002); Fernández-Huerga (2003); Hernanz (2003); Jimeno y Toharia (1993 y 1996); Polavieja (2006); Rodríguez-Gutiérrez (2003); Segura et al. (1991); Toharia (2005).

3 Por investigación cualitativa entenderemos aquella que no se basa en una muestra representativa de la población, sino que parte de un número limitado de individuos considerados "expertos" respecto al tema a tratar.

${ }^{4}$ No se ha descartado utilizar la información cualitativa para diseñar y ejecutar una segunda fase de investigación cuantitativa que permita generalizar los resultados.
} 
investigación cualitativa debe considerarse una primera parte de un trabajo científico completo, ya que sin lugar a dudas los resultados que se pueden obtener no son representativos de la población a la que pertenecen las personas entrevistadas. A pesar de ello, la investigación cualitativa presenta una clara utilidad, ya que permite, con presupuesto limitado, acceder a resultados que, posteriormente, se utilizan para diseñar de forma eficiente la segunda fase, cuantitativa, de la investigación basada en encuesta cerrada dirigida a una muestra representativa. Debe entenderse, pues, como un ejercicio de formulación de hipótesis que, posteriormente, deberían ser contrastadas con todo rigor.

La limitación del presupuesto limitó el estudio a la realización de 22 entrevistas, que se distribuyeron tal y como indica el Cuadro 1.

\section{Cuadro 1. Distribución de las entrevistas según CCAA y actividad económica ${ }^{5}$}

\begin{tabular}{ccccccc}
\hline & Constr. & $\begin{array}{c}\text { Hostelerí } \\
\text { a }\end{array}$ & $\begin{array}{c}\text { Ind. } \\
\text { Manuf. }\end{array}$ & Agric. & $\begin{array}{c}\text { Admón.. } \\
\text { Pub. }\end{array}$ & Total \\
\hline Cataluña & 1 & 1 & 4 & 0 & 1 & 7 \\
Andalucía & 0 & 1 & $2^{6}$ & 1 & 1 & 5 \\
Baleares & 1 & 1 & 1 & 0 & 0 & 3 \\
Madrid & 1 & 0 & 1 & 0 & 1 & 3 \\
Murcia & 1 & 1 & 1 & 1 & 0 & 4 \\
\hline Total & 4 & 4 & 9 & 2 & 3 & 22 \\
\hline
\end{tabular}

Se consideró oportuno, para garantizar la fiabilidad del resultado, punto siempre crucial cuando la investigación se limita al aspecto cualitativo, realizar personalmente todas las entrevistas. Se partía de un guión semiestructurado ${ }^{7}$ y la entrevista se adaptaba a las características del interlocutor.

Para establecer hipótesis de trabajo a validar en investigaciones

\footnotetext{
${ }^{5}$ Las entrevistas se realizaron entre los meses de enero a marzo de 2007.

${ }^{6}$ A pesar de que inicialmente se había programado realizar 1 entrevista a empresas del sector de la construcción y 1 entrevista a empresas del sector manufacturero, finalmente se realizó una entrevista a una empresa del sector eléctrico pero cuya labor principal es el montaje eléctrico en obra pública y privada. Dada su relación con el sector de la construcción se consideró una entrevista válida.

7 Diversos autores (ver, por ejemplo, Quivy y Campenhoudt (1997) o Strauss y Corbin (1998)) señalan la importancia de que el entrevistador o entrevistadora no formule demasiadas preguntas, ni que éstas sean demasiado concretas.
} 
posteriores, se ha utilizado como herramienta de clasificación de la información, un modelo por etapas. Los modelos por etapas tienen su origen en la psicología de la salud, y se han enfocado principalmente a estudiar el comportamiento preventivo. Un enfoque que se ha impuesto en los últimos años, con gran soporte teórico y empírico, es el de etapas propuesto por autores como Prochaska y DiClemente ${ }^{8}$ (1983), Weinstein (1988) o Kotler y Andreasen (1991).

\section{Presentación de las hipótesis de trabajo obtenidas}

Las entrevistas realizadas, en todos los casos a personas con responsabilidad directa de contratación dentro de la empresa $u$ organismo, fueron muy ricas en información, y permitieron establecer una serie de hipótesis de trabajo a validar en estudios posteriores, como era el objetivo de la investigación, y que se irán explicando a continuación:

\subsection{Hipótesis sobre usos y motivaciones del contrato temporal}

Inicialmente, en el proyecto de investigación y el cuestionario, se contemplaban dos apartados diferenciados para estos enfoques, considerados diferentes. Según los resultados de las dos entrevistas de prueba realizadas, y consecuente con una cierta estructuración de las decisiones de los agentes racionales, se esperaba obtener por un lado resultado sobre motivaciones, es decir qué es lo que lleva a una empresa a decidir usar un contrato temporal, y por otro lado sobre usos específicos, es decir, en qué puestos de trabajo o en qué circunstancias específicas, la empresa usa esos contratos. La realidad es que las personas entrevistadas tienen una única visión integrada en cuanto a motivaciones y usos lo que hace difícil distinguir uno de otro aspecto, por lo que finalmente se ha optado por agrupar los resultados en un único apartado, aunque se intentará distinguir lo que los propios entrevistados entendían por uso o por motivación.

$\mathrm{Al}$ ser preguntadas por el "uso" del contrato temporal, las respuestas obtenidas permiten distinguir tres diferentes situaciones, lo que nos permite formular unas primeras hipótesis. Estas tres situaciones son las siguientes:

- Empresas que tiene una actividad económica irregular, estacional o con puntas como puede ser la construcción, la agricultura y algunas manufactureras. En este grupo, el contrato temporal se utiliza

${ }^{8}$ El modelo de Prochaska y DiClemente también es conocido por modelo transteorético. 
para ajustar la carga del personal a los momentos de actividad, y poder descargarse cuando esta finaliza. Al no existir una actividad garantizada y continuada en el tiempo, el contrato temporal —en sentido amplio - se ve como la forma casi obligada de comportamiento. En el caso de las manufactureras, se cubren con contratos temporales las puntas de demanda de producción en fábrica y también tareas específicas como la descarga de contenedores o la limpieza de almacén. Es habitual, en este último caso, recurrir a las ETT.

- Empresas que tienen una actividad económica regular, como algunas de las empresas manufactureras y la Administración Pública. En este grupo, y siempre según las personas entrevistadas, el contrato temporal se utiliza a modo de "contrato prueba". Sólo una de las empresas entrevistadas manifestó utilizar el periodo de prueba correspondiente de un contrato indefinido. También puede ser utilizado para algún tipo específico de puesto de trabajo dentro de la empresa, como limpieza o para cubrir bajas por maternidad y similares. En este último caso, las empresas no utilizan el contrato temporal de forma habitual, excepto en los casos indicados y la razón suele ser que tienen plantilla fija y con poca variación. El caso de la Administración Pública es un caso aparte, ya que la temporalidad está muy relacionada con el hecho de que el contrato "indefinido" conllevaría la plaza de funcionario, y la salida a concurso de éstas está influida, no sólo por las necesidades laborales, sino también por las políticas de gasto de los diferentes entes.

- Empresas que tienen una actividad estacional previsible, como hostelería o agricultura. Cuando la actividad económica presenta estacionalidad, pero ésta es conocida por repetirse cíclicamente, las empresas pueden adaptarse a la misma utilizando el contrato de "fijo discontinuo". En todo caso, las empresas entrevistadas dijeron preferir dicho tipo de contrato al temporal.

Además, en 15 de las 22 entrevistas se manifestó una preferencia de uso del contrato temporal para puestos de trabajo de poca cualificación. Concretamente, en las empresas de sectores de actividad económica irregular como la construcción, los contratos fijos pertenecían a los "encargados", según palabras textuales de una de las personas entrevistadas: "Los fijos son los que llevan la obra; los oficiales y gente más cualificada. Se carga con estas personas aunque haya poco trabajo porque la puedes necesitar en un futuro. Acabas reteniendo a los que más dinero ganan”. Otra cita textual, correspondiente a una de las empresas entrevistadas del sector agrícola, apoya la línea desarrollada por la anterior, al afirmar: "Los fijos son nacionales, capataces, conocen la zona, el campo y el producto."

Incluso en aquellas empresas en las que la contratación temporal 
puede ser el paso previo a la incorporación a la plantilla de forma indefinida, si el lugar a cubrir era de alta cualificación, se optaba por la búsqueda del perfil idóneo mediante head hunters.

Se entrevistó a dos grandes empresas, del sector manufacturero pero de actividades económicas diferentes, que eran, cada una de ellas, líderes a nivel estatal y europeo. En una de ellas los contratos temporales se utilizaban exclusivamente en fábrica, para cubrir puntas de demanda. En la otra, los contratos temporales se utilizaban también en fábrica, pero en este caso la voluntad de la empresa era la de utilizar al máximo la temporalidad manteniéndose dentro de la legalidad vigente y de la no conflictividad interna de la empresa (acuerdos con el comité de empresa). La persona entrevistada explicaba la preferencia de la empresa por el contrato temporal por la vía de los costes y la poca especialización necesaria de los puestos de trabajo: "Sale barato. Los requisitos del personal, operarios, se forman en dos semanas. La persona no es importante."

Otra característica a destacar es la menor preferencia hacia los contratos temporales que tienen empresas que ofrecen servicios a clientes. Por ejemplo, de las empresas de hostelería entrevistadas, aquellas preocupadas por la atención al cliente preferían personal fijo (o fijo discontinuo caso de estacionalidad) porque la rotación de las personas perjudicaba la percepción que de la calidad del servicio tiene el cliente final. En el caso de un pequeño restaurante (4 trabajadores, además de los propietarios), se reconocía tener una clientela fija, la cual no se sentía a gusto, y se quejaba, si el personal rotaba con una cierta frecuencia. Pero también en el caso de una gran empresa de ámbito internacional, con cadena de hoteles y servicios de tour operador, se habían analizado las encuestas de satisfacción de los clientes, y se constataba que el nivel de satisfacción bajaba coincidiendo con la rotación de personal que provoca cambio en los equipos, tardando en recuperar los anteriores niveles unas dos semanas.

Por lo que hace referencia a las motivaciones, la información obtenida sugiere la existencia de tres características de las empresas que tienen influencia sobre las diferentes motivaciones transmitidas por las personas entrevistadas, y que se desarrollan a continuación.

Una primera característica sería el tipo de actividad económica, más allá del encasillamiento en un cierto sector productivo. Se han entrevistados empresas de sectores que habitualmente son citados como sectores de alta tasa de temporalidad, como por ejemplo la hostelería o la construcción, cuya motivación hacia el uso de ese tipo de contrato era escasa. En estos casos, existía una lógica hacia el uso prioritario del contrato indefinido basada en la regularidad de la demanda similar a la 
que podría existir en una empresa manufacturera. La hipótesis que se formula sería, entonces, que, bajo condiciones de regularidad de la actividad económica, se preferiría el uso del contrato indefinido, quedando el contrato temporal relegado a situaciones extraordinarias, como cubrir una baja por maternidad, o bien, como "contrato prueba" previa la contratación indefinida.

Una segunda característica sería la antigüedad de la empresa y la antigüedad media de su plantilla fija. Las empresas con decenas de años a sus espaldas, especialmente del sector manufacturero, podrían presentar una preferencia hacia el contrato temporal basado en experiencias propias que las han convertido en más adversas al riesgo de lo que la coyuntura económica justificaría. Así, uno de los entrevistados verbalizaba esta característica de la siguiente forma: "Nos encontramos con personal de cualificación baja haciendo tareas de almacén, con sueldos astronómicos. Le hablo de 300.000 pts. netas por quince pagas. La productividad de un sueldo tan alto no existe. Lo que podría ser una barrera de entrada para una empresa nueva que se incorporara a nuestro sector, es completamente al revés. Ellas contratan personal inmigrante o a sueldos muy bajos. (Son ellas las que) tienen una ventaja competitiva porque nosotros tenemos que asumir los trabajadores estos que hace tantos y tantos años que están (en la empresa)." Otra de las empresas que se encontraba en una situación prácticamente igual a la anterior, presentaba las mismas características: Pyme (entre 25 y 50 trabajadores), del sector manufacturero, con antigüedad superior a los 30 años (empresa y trabajadores), con trabajadores de sueldos elevados (por acumulación de antigüedad, subidas de convenio, etc.) en puestos de trabajo poco cualificados de baja productividad. La empresa los asume como coste fijo y, al mismo tiempo, aumenta su percepción del riesgo que conlleva un contrato indefinido. En cambio, en general (14 de las 22 entrevistas) las personas entrevistadas manifestaron ser conscientes de que, hoy en día, se puede despedir con la misma facilidad a una persona con contrato indefinido que con otro tipo de contrato. Los costes del despido, si la antigüedad del trabajador es poca, no se consideran importantes. Por ello, este freno a la contratación indefinida de este tipo de empresas "decanas" podría considerarse "irracional", ya que se basa en asimilar los costes de despedir a un trabajador de nueva incorporación con los costes de despedir a un trabajador con treinta años de antigüedad. Este comportamiento también se puede interpretar desde la perspectiva de la ventaja comparativa: las empresas nuevas, con menores costes, representan una amenaza competitiva para empresas con tradición que no pueden capitalizar su "gestión del conocimiento" al pertenecer a 
sectores en los que no hay demasiada acumulación de capital humano, o bien en los que las condiciones de producción han cambiado mucho en los últimos años ${ }^{9}$. Bajo estas circunstancias, la temporalidad se percibe como “(...) una solución, no un problema”, ya que les permite adaptarse a las nuevas circunstancias tanto productivas como competitivas, alejándose de un modelo de relaciones laborales que ya les ha fallado a lo largo de su historia como empresa.

La tercera característica que parece influir en el uso del contrato temporal es la percepción que tenían las personas entrevistadas de que el mercado de trabajo español se define, hoy en día, por la falta de trabajadores que respondan al nivel de cualificación que la empresa necesita. La percepción era independiente del sector -excepto parcialmente para la Administración Pública - y del puesto de trabajo, incluso si éste responde a niveles bajos de cualificación. Prácticamente todas las personas entrevistadas (20 de los 22 casos) coinciden en señalar como principal problema en el día a día de la empresa, en relación al mercado laboral, las dificultades para encontrar mano de obra. Frases como "no se encuentra gente", "Falta mano de obra, es un problema muy acuciante.", "aquí no se encuentran personas del perfil que necesitamos", "cuesta encontrar personas realmente cualificadas", "tenemos puntas de necesidad de personal, y no lo hay, nadie quiere trabajar en el campo", "problema: muy poca mano de obra", "no hay profesionales y cuestan de fidelizar", "faltan profesionales, cuando llegan al puesto no son profesionales", "la mano artesana ha desaparecido", "el principal problema es la dificultad en encontrar mano de obra, la que hay es poco cualificada", "(el principal problema es) encontrar personas con capacidad y actitudes, predispuestas", "la gente no está suficientemente formada, y los jóvenes menos", "nos cuesta encontrar personal porque hay mucha derivación de personal cualificado hacia la construcción por mejores salarios", "el campo nadie lo quiere", "el sueño de la vida de nadie no es ser camarero", "hay mucho trabajo y poca gente buena", reflejan claramente la opinión mayoritaria de los responsables de contratación entrevistados. Este hecho parece influirles de dos formas que a priori parecen opuestas. En primer lugar, ante el temor a que el trabajador de nueva contratación no cubra adecuadamente las necesidades de la empresa, se tiende a ofrecer, en primera instancia, un contrato temporal ${ }^{10}$. Me refiero a

9 Este último era el caso de una de las dos empresas citadas, que empezó su andadura con un producto cuyo proceso de fabricación era mecánico y en la actualidad es electrónico.

10 Se encontró que la práctica más común era la de ofrecer contrato temporal durante un mínimo de 6 meses, por ejemplo dos contratos de 3 meses, antes de ofrecer el 
"necesidades de la empresa" más que a "cualificación laboral" porque en algunos casos (6 de 22) señalaban no sólo la falta de formación o de experiencia, sino también la falta de predisposición a aceptar horarios inconvenientes o movilidad geográfica, aunque ésta sea de forma puntual. Al profundizar en estas percepciones los entrevistados, 14 de las 22 personas, consideraban que el problema era más grave cuando se buscaban trabajadores entre la población joven autóctona, tanto a nivel de actitud (poca predisposición a aceptar condiciones laborales "incómodas"), como de formación (falta de adecuación de los estudios, principalmente de FP, a las demandas de la empresa).

Una de las explicaciones que los propios entrevistados daban libremente (respuesta espontánea) fue que la buena situación de la economía española alimenta este comportamiento: los trabajadores, en especial los jóvenes, pueden encontrar trabajo; si se acaba un contrato, no tienen grandes dificultades en conseguir otro, por ello dejan de valorar la seguridad de un contrato indefinido a cambio de repetir un ciclo compuesto de periodos de trabajo con contrato temporal seguidos de periodos de cobro de la prestación por desempleo que, en algunos casos, puede simultanearse con economía sumergida ${ }^{11}$.

En segundo lugar, la apreciación de la falta de mano de obra, y especialmente mano de obra de cualificación media ${ }^{12}$ — técnicos, operarios de nivel medio, montadores, electricistas, etc.-, provoca que, cuando un trabajador responde a las expectativas de la empresa, ésta no dude en actuar para asegurarse de su permanencia en ella. Esta actitud comporta, por un lado el ofrecimiento de un contrato indefinido, aunque las personas entrevistadas reconocen que este tipo de contrato parece haber perdido fuerza como incentivo para el trabajador, y por otro lado, el posible sobredimensionamiento de la plantilla, que afecta especialmente y de forma puntual a empresas con actividad económica irregular — construcción, por ejemplo-: "Ios buenos te cargas con ellos, al no haber gente cualificada tienes que conservarlos aunque no tengas trabajo, porque los puedes necesitar en el futuro, hay que retenerlos y

contrato indefinido.

${ }^{11}$ Con todo el cuidado con el que hay que tomar siempre la investigación cualitativa, es de destacar que 5 de las personas entrevistadas, de diferentes comunidades autónomas y sectores, coincidieron en señalar que se suele combinar el cobro de la prestación por desempleo con las "chapuzas”(literal).

12 Para puestos de trabajo de alta cualificación - I+D, altos cargos de gerencia, directores de área, etc.- parece haber coincidencia en la opinión de que cuesta menos encontrar las personas que los cubran, aunque existe la queja de que en ocasiones se tiene que recurrir a head hunters de implantación paneuropea para asegurar el nivel idiomático (inglés o alemán) de las personas. 
hacerlos fijos". También puede afectar a la política salarial, en 10 de los 22 casos (19, si descontamos las 3 entrevistas de la Administración Pública), se reconoció pagar por encima de convenio como forma de fidelización: "pagamos por encima de convenio, tenemos categorías duplicadas y la gratificación se establece en función de la asunción de compromisos", "pedimos actitud, superar el training; ofrecemos elevar la categoría y tendrán un extra con respecto a los beneficios", "tenemos problemas para fidelizar a los empleados, estamos mejorando mucho la oferta económica y ofrecemos otras cosas, el treinta por ciento del salario es en especie", "el problema de no encontrar gente lo solventamos ofreciendo gratificaciones", "el convenio marca 5,81 la hora, pero se paga más alto, se pueden sacar 1200 al mes fácilmente".

Si aceptamos la visión de la problemática de las personas entrevistadas, el uso del contrato temporal respondería a un cálculo racional con respecto a la productividad del trabajador: si el trabajador "no supera" el periodo de prueba, finaliza el contrato y la empresa no se lo renueva, mientras que al trabajador que sí cumple con las expectativas de la empresa se le ofrece el contrato indefinido. Las renovaciones del contrato temporal de un trabajador productivo (uno que acabará pasando a contrato indefinido en la misma empresa) responderían a la costumbre de aprovechar la flexibilidad que ofrece la legislación, especialmente en empresas con aversión al riesgo, como se comentaba anteriormente, pero el objetivo final es la conversión a fijo "si él (el trabajador) quiere, claro". Estas empresas tienen, de hecho, un comportamiento muy parecido al que pretende impulsar la última reforma laboral, aunque la mayoría de las entrevistadas no solía utilizar el máximo de renovaciones que la ley permitía, y por tanto esperaríamos que sus decisiones actuales no se vieran influidas por dicha reforma. Por otro lado, las personas con un historial profesional de contratos temporales, encadenados o no, en diferentes empresas, podrían ser vistos como trabajadores poco productivos, por lo que las empresas podrían ser reacias a ofrecerles un contrato indefinido, así se habría caído en un círculo vicioso en el que la temporalidad podría engendrar nueva temporalidad, y en un mercado de información asimétrica, el historial de contratos temporales actuaría como señal informativa para el empleador.

Finalmente destacar dos casos, ambos del sector manufacturero, que aducían como causa principal al uso de la contratación temporal el ahorro de costes que les representa. En uno de los dos casos, la empresa era una pyme (25-50 trabajadores) y consideraba una necesidad el uso de la contratación temporal: "utilizamos la temporalidad en la medida en que nos dejan por ahorro de costes, (...) nosotros, ahora, no 
podríamos subsistir sin la subcontratación o los trabajadores que nos envía la ETT (...) si todo volviera a ser rígido tendríamos que cerrar”. El otro caso se trataba de una gran empresa, líder europea en su sector, con 1200 trabajadores y que consideraba el uso de la contratación temporal vía ETT como absolutamente racional en función del ahorro de costes que le representaba: "Cada vez es mucho más barato y a las grandes empresas con nuestros volúmenes de facturación con las ETT y las condiciones que nos están poniendo..., es que nos sale muy barato; ellas asumen el gasto de la ropa, el gasto en seguridad, un rápel de formación, asumen el absentismo... es que me sale muy barato". Es importante quizá matizar que también en ambos casos los contratos temporales se utilizaban para personal de producción en fábrica o para tareas de muy baja cualificación (descarga de contenedores, limpieza).

Sintetizando la información anterior, las hipótesis sugeridas que se deberían contrastar son las siguientes:

- Primera, el contrato temporal se utiliza como forma de adaptación al mercado, y en este sentido vendrá influido por características como: nivel de globalización del mercado, concentración empresarial, competencia, elasticidad de la demanda,...

- Segunda, el contrato temporal se utiliza en la actualidad como forma de "contrato prueba", paso previo a la contratación indefinida. La sensación de falta de mano de obra profesional o de las cualificaciones que la empresa necesita, apoya la necesidad por parte de las empresas de este periodo de prueba, para confirmar mediante la experiencia la idoneidad de la persona. El contrato indefinido (la oferta del mismo al trabajador) se convertiría en un elemento de fidelización para las personas que "superan la prueba".

- Tercera, en relación con la anterior hipótesis, el contrato temporal permanente (es decir, el que no se convierte en indefinido) quedaría entonces relegado para los puestos de trabajo de menor cualificación.

- Cuarta, por otro lado, la información obtenida, teniendo en cuenta que es la visión de los empleadores, vendría a sugerir que cuando existe bonanza económica, la facilidad de encontrar trabajo alimenta la existencia de temporalidad debido a la menor predisposición del empleado a aceptar contratos indefinidos.

- Quinta, independientemente del nivel de cualificación, si el puesto de trabajo requiere del contacto directo con el cliente, los empleadores prefieren que no haya rotación de personal, para así mejorar el nivel de satisfacción del cliente. En esas circunstancias, preferirían usar contratos fijos a temporales.

- Sexta, las empresas con actividad económica regular sienten 
una motivación menor hacia el uso del contrato temporal, independientemente del sector productivo al que pertenecen.

- Séptima, la antigüedad de la empresa y la antigüedad media de su plantilla fija puede influir en la motivación al uso del contrato temporal: a mayor antigüedad, mayor motivación, especialmente en el sector manufacturero. Esta hipótesis nos indicaría la existencia de una gran aversión al riesgo debido a experiencias negativas anteriores en esas empresas.

\subsection{Hipótesis sobre la actitud de los empleadores hacia el fenómeno de la temporalidad}

Como ya se ha comentado anteriormente, los modelos por etapas tienen su origen en la psicología de la salud, y se han enfocado principalmente a estudiar el comportamiento preventivo. A pesar de que su origen sea éste, dichos modelos pueden aplicarse a cualquier aspecto en el que exista un problema (sea salud, de riesgo o de cualquier otro tipo) que pueda afectar a los individuos analizados, y que la actitud y el comportamiento de las personas pueda influir en la ocurrencia del riesgo. Para poder analizar mediante el modelo un cierto comportamiento o actitud se debe partir de la hipótesis de que existe una cierta problemática respecto a la cual se desea posicionar a los individuos. En este caso dicha problemática es el fenómeno de la temporalidad, y la hipótesis de partida se podría enunciar como sigue: "La temporalidad es un problema porque, a medio y largo plazo, puede ocasionar pérdidas de acumulación de capital humano con la consiguiente merma en productividad". Partimos, por tanto, de la premisa de que "la temporalidad es un problema", pero también sabemos que es un problema del que las empresas pueden "protegerse", estableciendo otros tipos de relación laboral. Para hacer efectiva la protección, las empresas deben estar incentivadas a ello, sea por un convencimiento propio $-\mathrm{y}$ éste es siempre el mejor incentivo-, sea porque las medidas que desde las diferentes administraciones se implementen les muevan a un cambio de comportamiento. El modelo por etapas lo que pretende es averiguar cuál es la actitud de los individuos para de esta forma establecer cuál es la mejor medida que puede provocar ese cambio de comportamiento. Por ejemplo, si la persona desconoce que la temporalidad puede representar un problema a medio largo plazo por sus posibles efectos negativos sobre la productividad, entonces medidas basadas en la comunicación tenderán a ser efectivas. En cambio no lo serán en absoluto si el responsable en contratación ya es consciente de ese hecho, pero su cálculo coste - 
beneficio le lleva a concluir que su empresa, individualmente, no se ve perjudicada por ese aspecto. Los mecanismos de incentivos son complejos de diseñar, en ocasiones caros de implementar y suelen tener efectos secundarios no previstos, por lo cual mientras mayor sea el volumen de información disponible, más eficiente podrá ser el diseño y aplicación de los mismos.

Dado el alcance de este documento, como notas de investigación, y para no alargarlo excesivamente, se comentarán directamente las hipótesis sobre actitudes que sugiere la aplicación del modelo por etapas para clasificar las entrevistas realizadas.

El modelo por etapas aplicado a la clasificación de las entrevistas realizadas sugiere como hipótesis de trabajo (a contrastar mediante investigación con muestra representativa) que el comportamiento de los empleadores hacia el fenómeno de la temporalidad es un comportamiento racional, influido por la idiosincrasia particular del sector de actividad y por las características globales de la economía española en su vertiente productiva.

La racionalidad de sus decisiones se desprende de los argumentos expresados, tanto por aquellas empresas que consideran que la temporalidad no es un problema, como por las que sí lo creen de ese modo. En todo caso debería matizarse esta idea en el contexto del mercado de información asimétrica y de una aversión al riesgo elevada para algunas empresas en particular, de largo historial productivo y que han sido perjudicadas en algunas de sus etapas por rigideces anteriores del mercado laboral. En este sentido, no es de extrañar que la mayoría de las empresas que responden a este perfil sean del sector manufacturero, el más castigado por crisis económicas anteriores, pero también por el proceso de globalización de la economía y el aumento de la competencia internacional. Es interesante también la constatación de que para las grandes empresas manufactureras, el ahorro en costes que supone la temporalidad no se centra tanto en los costes del despido, sino en aquellos otros costes que, al gestionar la contratación a través de las ETT, son asumidos por estas, en particular el absentismo. No parece que, para estas empresas, la Ley de Reforma del Mercado Laboral vaya a impactar de forma importante. Para las de mayor tamaño, los pactos internos con el Comité de Empresa pueden propiciar la contención del uso de los contratos temporales. En este caso, la empresa lo racionalizaría como un ahorro en costes por reducción de la conflictividad interna.

En cuanto a la idiosincrasia particular del sector de actividad económica de la empresa, las entrevistas han permitido constatar que en la construcción, en ciertos subsectores de la hostelería y en la 
agricultura las empresas tienen dificultades para poder llevar a cabo su decisión de limitar al máximo la rotación de su personal. Además estas son actividades que están siendo "abandonadas" por la población autóctona, creándose un exceso de demanda que se cubre con mano de obra de origen extranjero. Como indicaba uno de los entrevistados: "Este sector es un sector de paso para los trabajadores. La realidad es que la gente quiere tener dinero y tiempo para gastarlo y en este sector siempre va a faltar tiempo (...) después del éxodo del sector agrícola va a venir el éxodo del sector de la hostelería”. La falta de integración social del colectivo de trabajadores extranjeros; su preferencia clara hacia salarios, antes que hacia condiciones laborales, o su desconocimiento de la legislación vigente en el marco laboral provocan conflictos añadidos que acentúan la problemática. Las empresas que se encuentran en esta situación no se ven afectadas por la reforma del mercado, ya que son ellas las primeras interesadas en disponer de herramientas contractuales que les permitan conservar el mismo equipo de personas, si éstas le funcionan. Allá donde es posible, se aplica el contrato de fijo discontinuo, o incluso las empresas prefieren sobredimensionar ligeramente su plantilla — con respecto al volumen de trabajo en un momento determinado - a perder trabajadores productivos. Estas empresas coinciden en sentirse poco apoyadas por la administración, por lo que un análisis en detalle que permitiera particularizar la norma general para aplicarla a su caso específico sería deseable. Como verbalizaban algunas de las personas entrevistadas: "Entra y sale gente eventual, pero no nos gusta, tanto movimiento da mucho gasto administrativo y siempre les tienes que estar enseñando, pero son circunstancias del trabajo y hay que lidiar con ellas", "El personal eventual tendría que ser indefinido, pero no les interesa, el seguro agrario no les compensa, se jubilan con una miseria, el campo es sólo un complemento", "No nos interesa la rotación de trabajadores, hay que enseñarles y se pierde tiempo y dinero, pero no les motiva el contrato fijo, prefieren dedicarse a la chapuza", "Desde la administración me dicen que algo falla en la empresa, pero en la última regularización de 78 contratos sólo me quedan 8 , todo son bajas voluntarias, no tienen continuidad, la rotación es inevitable”.

Las características globales del modelo de crecimiento español sería el último elemento hipotéticamente explicativo de las actitudes de las personas entrevistadas hacia la temporalidad. El crecimiento de la economía española ha estado dirigido en los últimos años por sectores que padecen de la dificultad citada anteriormente para conseguir niveles bajos de rotación de sus trabajadores. En muchos casos se citaba la "competencia" de los sueldos ofrecidos por el sector de la construcción 
como principal obstáculo para conseguir mano de obra para empresas de otros sectores, como la hostelería o incluso el sector manufacturero. Por otro lado, no parece destacar el crecimiento de aquellos sectores económicos en los que más importante es el buen uso del capital humano. El sistema productivo español debería cambiar más rápidamente de lo que lo está haciendo hacia esas producciones de alto valor añadido, basadas en procesos de innovación, que den incentivos a las empresas para apostar por la acumulación de capital humano. Mientras, como decía uno de los entrevistados, "la persona no sea importante" va a ser difícil que se generen los incentivos correctos que permitan el cambio de actitud y de comportamiento hacia el problema de la temporalidad.

Finalmente recordar que, a lo largo de las entrevistas, se ha detectado lo que podríamos considerar una desconfianza de las personas responsables de la contratación hacia los jóvenes, en dos aspectos: la validez de su formación por un lado y la actitud hacia el trabajo por otro.

\section{Conclusiones}

Aun considerando las limitaciones consustanciales a una investigación de tipo cualitativo basada en entrevistas personales, los resultados mostrados revisten el suficiente interés y sobretodo dejan preguntas abiertas y atractivas de responder, especialmente por lo que representa la interpretación de los hechos recopilados con la idea de la existencia de una cierta "cultura de la temporalidad". El comportamiento y las actitudes detectadas en algunos casos lo son "a pesar de" el responsable de la contratación, mientras que en otros casos no podemos considerarlos ajenos a procesos de globalización de los mercados de productos que inciden en el mercado laboral. La adaptación de nuestra industria a estas nuevas condiciones de mercado quizá haya encontrado un "flotador" en el contrato temporal, pero éste no debería ser utilizado como mecanismo para reducir los costes laborales, ya que por un lado provoca una segmentación de nuestro mercado laboral, pero por otro su existencia actúa en contra de los incentivos que nuestras empresas deberían tener para adaptarse a este nuevo entorno, no por la vía de la reducción en costes, sino por la vía de la especialización en productos y servicios con un uso mayor de capital humano, dentro del proceso de adaptación a la Sociedad del Conocimiento. Así, la problemática de la temporalidad parece englobarse dentro de una problemática mayor, que en los últimos 
tiempos puede haber quedado escondida por los buenos resultados de otros sectores económicos de gran crecimiento, como la construcción. Esta hipótesis de trabajo, caso de confirmarse - para lo cual se requeriría realizar el correspondiente estudio cuantitativo- nos daría información interesante y útil para el diseño de mecanismos de incentivos adaptados tanto a la realidad de nuestro mercado laboral como a la realidad empresarial.

\section{Bibliografía}

Aguirregabiria, V. y Alonso-Borrego, C. (1999): "Labor Contracts and Flexibility: Evidence from Labor Market Reform in Spain”, Documento de trabajo SSRN: http://ssrn.com/abstract=156451.

Bandura, A. (1977): "Self-efficacy: Toward a unifying theory of behavior change". Psychological Review, 84, pp. 191-215.

Bandura, A. (1982): "Self-efficacy mechanism in human agency". American Psychologist, 37, pp. 122-147.

Dolado, J.J., García - Serrano, C. y Jimeno, J.F. (2002): "Drawing Lessons from the Boom of Temporary Jobs in Spain". Economic Journal (2002), 112, pp. 270-295.

Fernández - Huerga, E. (2003): “Causa de la utilización del empleo temporal y la subcontratación: el caso de las industrias extractivas en León”. Mimeo. Departamento de Economía. Universidad de León.

Hernanz, V. (2003), El trabajo temporal y la segmentación: un estudio de las transiciones laborales, Consejo Económico y Social, Madrid.

Jimeno, J.F. y Toharia, L. (1993), "The effects of fixed-term employment on wages: Theory and evidence from Spain", Investigaciones Económicas, 17 (3), pp. 475-494.

Jimeno, J.F. y Toharia, L. (1996), "Effort, absenteeism and fixed term employment contracts", Revista Española de Economía, 13(1), pp. 105119.

Kotler, Philip y Alan Andreasen, (1991), Strategic Marketing for Non Profit Organizations. Prentice Hall.

OCDE (2007), Job for Youths: Spain. OECD Publications. (http://213.253.134.43/oecd/pdfs/browseit/8107081E.PDF )

OCDE (2007), Job for Youths: Spain (resumen en español). OECD Publications. (http://www.oecd.org/dataoecd/14/38/38461194.pdf) 
Polavieja, J.G. (2006), "The Incidence of Temporary Employment in Advanced Economies: Why is Spain Different?", European Sociological Review, 22(1), pp. 61-78.

Portell, M y Solé M.D. (1999): Riesgo percibido: un procedimiento de evaluación. NTP 578. MTAS, 1999.

Prochaska, J. O., DiClemente, C. C., y Norcross, J. C. (1992): "In search of how people change”. American Psychologist, 47, pp. 1102-1114.

Prochaska, J. O., y DiClemente, C. C. (1983): "Stages and processes of self-change of smoking: Toward an integrative model of change". Journal of Consulting and Clinical Psychology, 51, pp. 390-395.

Quivy, R. y Van Campenhoudt, L. (1997): Manual de Recerca en Ciències Socials. Herder.

Rodríguez-Gutiérrez, César (2003): “Una explicación de los cambios recientes en la tasa de temporalidad de las empresas manufactureras españolas". Presentado en las V Jornadas de Economía Laboral, Reus, 2003.

Segura, J., Durán, F., Toharia, L. y Bentolila, S. (1991): Análisis de la Contratación Temporal en España. Colección Economía y Sociología del Trabajo. Ministerio de Trabajo y Seguridad Social.

Strauss, A. y Corbin, J. (1998): Basics of Qualitative Research. Sage Publications Inc. Thousand Oaks.

Toharia, L. (dir.) (2005): El problema de la temporalidad en España: un diagnóstico. Ministerio de Trabajo y Asuntos Sociales, Madrid.

Velicer, W. F., DiClemente, C. C., Prochaska, J. O., y Brandenburg N. (1985) "Decisional balance measure for assessing and predicting smoking status". Journal of Personality and Social Psychology, 48, pp. 1279-1289.

Velicer, W. F., DiClemente, C. C., Rossi, J. S., y Prochaska, J. O. (1990): "Relapse situations and self-efficacy: An integrative model". Addictive Behaviors, 15, pp. 271-283.

Weinstein, N.D., (1998): "Stage theories of health behaviour". Health Psychology, 17, pp. 290-299. 